\title{
LUMINOUS CONTENT OF GALAXIES: INFERENCES FROM SUPERNOVAE
}

\author{
J. Craig Wheeler \\ Department of Astronomy \\ University of Texas
}

\begin{abstract}
Some luminous stars undoubtedly explode as supernovae, but it is not yet certain that fate awaits them all. The connection between luminous stars and supernovae is reviewed in terms of the statistical rates of supernovae and pulsars, the constraints of nucleosynthesis, and the various classifications of supernovae by their spectra and light curves, including a newly confirmed class of peculiar Type I supernovae.
\end{abstract}

\section{Introduction}

There are a few simple statements about supernovae that reflect the common understanding of them. The rate of explosion of Type I supernovae (SNI) is about the same as that of Type II supernovae (SNII) in our Galaxy. The rate of explosion of SNII is about the same as the birthrate of stars with mass in excess of $10 \mathrm{M}_{\odot}$ and of the birthrate of pulsars. The Crab nebula is an obvious example of a supernova which produced a pulsar. Massive stars account for the synthesis of the heavy elements.

Based on these popular notions, one might conclude that all stars with $M>10 \mathrm{M}_{\odot}$ explode as SNII, produce pulsars, and generate the heavy elements. There are numerous unanswered questions, however, that suggest that few of these issues are settled. The evolution of massive stars will be briefly summarized in Section 2 to set the stage. The information, uncertainties and open questions involved with the study of supernova statistics in various galaxy types will be addressed in Section 3. The constraints from nucleosynthesis are discussed in Section 4, and the different categories of supernovae classified by spectra and light curves and some speculations on those differences are presented in Section 5.

\section{Evolution}

Figure 1 gives a summary in the $\log \rho_{c}, \log T_{c}$ plane of the evolution of massive stars. Stars with mass less than about $8 \mathrm{M}_{\odot}$ form dense, degenerate carbon/oxygen cores. If they proceed to carbon ignition, they are believed to explode by means of a subsonic deflagration process (Mazurek, Meier, and Wheeler 1977) and leave no compact remnant. Most of these stars, however, are destined to eject their envelopes as planetary nebulae 


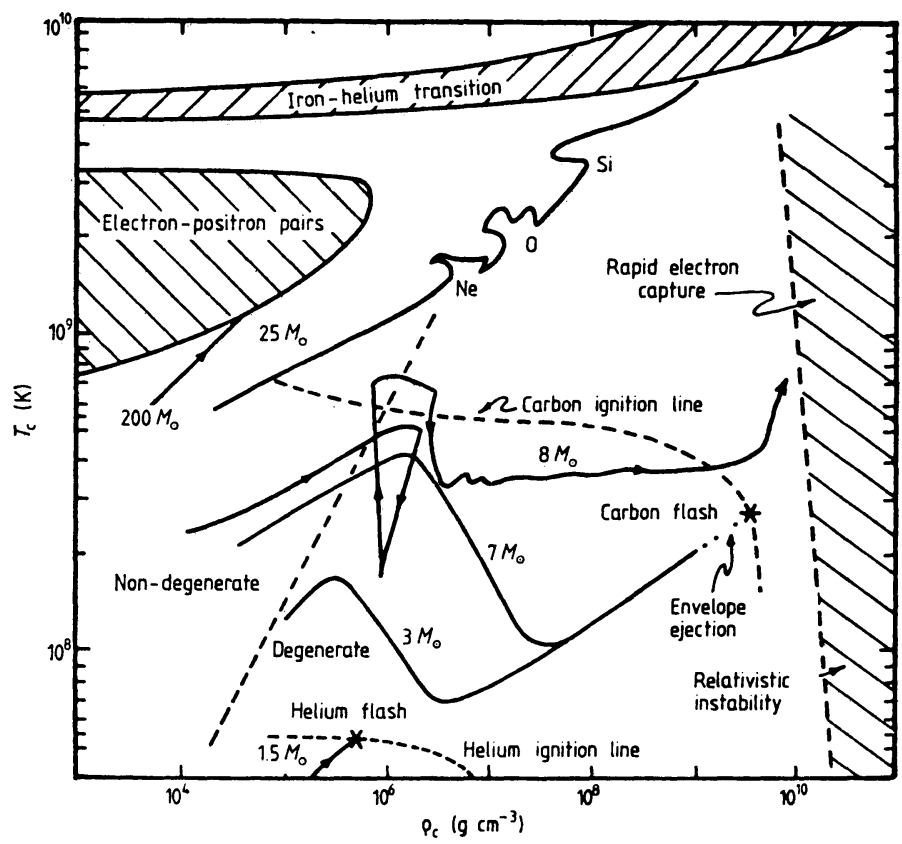

Figure 1. Evolutionary tracks are given in the plane of central density and central temperature. Most stars with mass less than about 8 solar masses produce white dwarfs after envelope ejection, but some may explode by degenerate carbon deflagration. Above 8 solar masses most stars are predicted to undergo core collapse. (From Wheeler 1981).

and leave the cores to from cooling stable white dwarfs. We do not know if some fraction of these stars near the upper mass limit retain their envelopes and proceed to explode. The currently most popular model for SNI involves the delayed deflagration of such a $\mathrm{C} / \mathrm{O}$ core left behind in the first stage of evolution which is later rejuvenated by mass transfer from a companion (Sutherland and Wheeler 1984).

Evolutionary models of stars with mass just in excess of about $8 \mathrm{M}_{\odot}$ up to about $10-12 \mathrm{M}_{\odot}$ show that carbon ignites off-center and burns non-degenerately. A degenerate core of $\mathrm{O} / \mathrm{Ne} / \mathrm{Mg}$ forms and collapses from the effects of rapid electron capture (Nomoto 1984). The resulting collapse is hindered, but not reversed by oxygen burning. This and the steep density profile which accelerates the shock enhance the probability that a rebound shock from neutron star formation will generate an explosion (Hillebrandt, Nomoto, and Wolff 1984).

Above $10-12 \mathrm{M}_{\odot}$, all burning occurs quasistatically and the star forms an iron core. This core photodisintegrates and collapses. There is considerable question whether an explosion ensues in this case by the direct action of a bounce shock, but heating by neutrinos leaking from the neutronized core on timescales approaching 1 second may boost a standing, stagnated shock into an outward propagating explosion (Wilson 1984). 
Stars of this mass, which are thought to be prime candidates for the synthesis of heavy elements, may explode, but some may collapse to produce black holes.

Masses in excess of about $100 \mathrm{M}_{\odot}$ are thought to collapse due to the formation of electron/positron pairs. This collapse is reversed by oxygen ignition and leads to total disruption unless the mass exceeds about $300 \mathrm{M}_{\odot}$, in which case the collapse proceeds to completion resulting in the formation of a black hole (Wheeler 1977, Woosley and Weaver 1982).

\section{Statistics}

Tammann (1982) gives the rate of explosion of SNI and SNII in the Galaxy as 1 per 36 years and 1 per 44 years, respectively, based on interpolation from extragalactic rates. A comparison of the rate of occurrence of SNII with the birthrate of massive stars (Wheeler 1981) shows that if stars with mass significantly less than $10 \mathrm{M}_{\odot}$ explode, there would be more SNII than observed. On the other hand, statistics do not determine whether stars with mass much in excess of $20 \mathrm{M}_{\odot}$ explode, because such explosions are expected to be rare and lost in the noise. Nucleosynthesis provides some constraint on these massive stars, as will be argued in section 4.

Figure 2 shows that there are uncertainties in the ratio of SNI to SNII in spiral galaxies. This figure presents raw number counts from the magnitude limited sample of Oemler and Tinsley (1979). One cannot prove, based on this data, that the ratio of SNI to SNII is not unity, but neither is there a strong basis for assuming that in our Galaxy (Sbc) that the ratio is unity. The net supernova rate can also be estimated from the occurrence of historical supernovae. Breaking down the rate by type is more difficult. Several are suspected of being SNI, but there is no known SNII in that sample. The Crab nebula event might have been a SNII, but its light curve is ill determined, and it has many features which are simply unorthodox, and difficult to categorize. In any case, despite these uncertainties, there is little evidence which would support the notion that SNII derive from stars with original mass much less than $10 \mathrm{M}_{\odot}$.

The rate of pulsar formation, assuming only about 20 percent are observed due to beaming, is about one per 20-50 years (Lyne 1982). This rate is crudely consistent with the rate of formation of SNII. A troubling fact is that evidence for neutron stars is observed only in filled center or composite type supernova remnants (SNR), not in the shell- type remnants which are 3/4 of the sample (Helfand 1984a). With the advent of the Einstein Observatory this search can be made not only in the radio which may be beamed, but by searching for X-ray synchrotron nebulae which should be isotropic emitters. Since the rate of formation of filled shell remnants which show evidence for pulsars is low, and the majority of SNR show no such evidence, the question is raised as to whether there must be a source of pulsars with no associated supernova remnant.

Helfand (1984b) has raised another interesting possibility. He questions whether the pulsar radiation is, in fact, not beamed, so the directly observed rate, about one pulsar per 200 years, which agrees with the rate of formation of filled center SNR, is the appropriate one. By this hypothesis, pulsars would be produced in all, but only in, the filled center SNR. The shell type SNR would be supposed to come from some event, perhaps SNI, which left no neutron star.

Another view on this problem is provided by a statistical study of the angular distribution of SNR ( $\mathrm{Li}$, Wheeler, and Bash, 1984; Li, Wheeler, Bash, and Jefferys 1985). These studies examine the distance independent angular distribution of the SNR as compared to Monte Carlo models which vary the proportion of SNR in the spiral arms and 


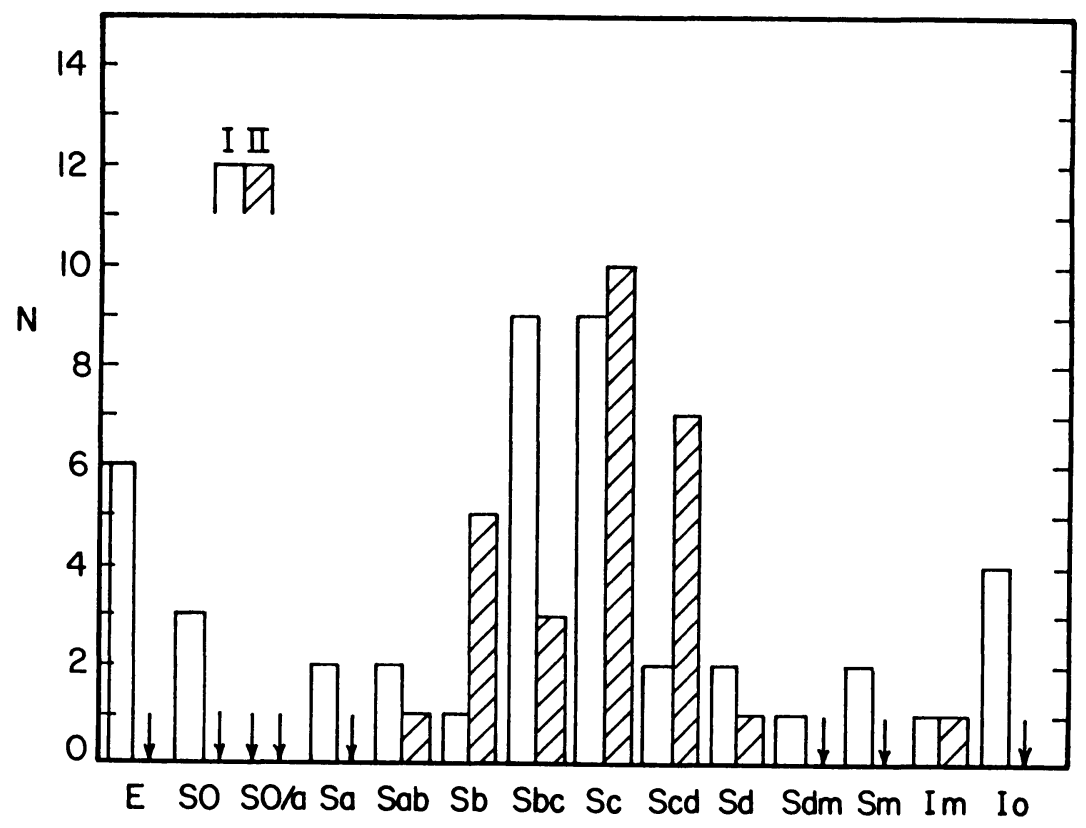

Figure 2. The number of supernovae identified from their spectra as SNI or SNII is presented as a functionof galaxy type from the magnitude limited sample of Oemler and Tinsley (1979). Two of the supernovae in the IO category were in NGC 5253 which should perhaps be reclassified as Im. (From Wheeler and Wheeler 1983).

the exponential disk. These studies reject the hypothesis that the shell type SNR are a disk population as would be expected if they were entirely from SNI. The model which best fits the data is one in which all the SNR reside in spiral arms, implying that they had massive star progenitors. The questions of why SNI do not seem to contribute to the sample of old SNR (perhaps because with large scale height they expell their ejecta from the galaxy) and why the shell SNR show no sign of pulsars remain open.

Models of SNII light curves, particularly the type with a distinct plateau (Falk and Arnett 1977; Litvinova and Nadyozhin 1983), can be reproduced with an explosion in an extended envelope. This strongly suggests that such supernovae are not merely massive stars, but likely to be red supergiants. An interesting question then arises as to why there is an apparent paucity of such events in late-type galaxies.

Figure 2 shows the extreme example of this phenomenon are the $\mathrm{I} 0$ galaxies. As for elliptical galaxies, these galaxies have been observed to have only SNI, no SNII. For ellipticals the standard argument is that there is no recent star formation, so all the supernovae must be old, but I0 galaxies typically have dark dust lanes, a typical sign of on-going star formation. Oemler and Tinsley (1979) have used such evidence to argue that SNI come from intermediate mass stars and that ellipticals must have a current level of star formation. An interesting example of a galaxy which is typically, if not completely accurately, labeled an I0 is M82, a site of particularly active star formation which 
apparently has generated a number of deeply buried radio SNR (Kronberg et al 1985). The type of these events is, of course, unknown.

The paucity of SNII is not complete in other late type galaxies, but perhaps just as mystifying. Oemler and Tinsley present rates for galaxies of different morphological type corrected for various selection effects. For Sc and Scd galaxies, the most prolific supernova producers by number, they give the rates of SNI and SNII as 0.40 and 0.35 , respectively, in units of $10^{12} \mathrm{~L} \odot-y r$. For the category including Sd, Sdm, Sm, and Im they give 0.48 and 0.14 in the same units, showing the marked paucity of SNII. They give 1.7 and 0 for $\mathrm{IO}$ galaxies. The rate of production of SNI in $\mathrm{IO}$ galaxies is based on only four supernovae, the two events in NGC 5253, SN1985b and SN1972e and two others. Campbell and Terlevich (1984) have shown, however, that NGC 5253 has an abundance of red supergiants and it is probably nearer to an Im in classification, than an I0. Thus the rate of SNI in I0 galaxies should be only 0.8 based on only two objects, and hence uncertain, although interestingly large. The rate of SNI in the Sd -Im category should probably likewise be increased slightly, enhancing the relative paucity of SNII. The Sd through Im galaxies are characterized by abundant star formation, $O$ stars, and red supergiants. Where, then, are the SNII? Are they hidden by dust as in M82? Is the paucity a temporary thing, caused by observing a starburst at the wrong phase? Does bimodel star formation short change some galaxies of massive stars? These questions are worthy of serious investigation.

\section{Nucleosynthesis}

An important development in the long study of nucleosynthesis of the heavy elements in massive stars has been the recent upward revision of the rate of the ${ }^{12} \mathrm{C}(\alpha, \gamma){ }^{16} \mathrm{O}$ reaction by a factor of 3 to 5 (Kettner et al 1982). This revision changes the systematics all along the alpha chain and gives a particularly satisfying fit of the isotopic and elemental ratios to the cosmic values for elements from carbon to the iron peak in the model of a star of 25 $M_{0}$ (Woosley 1985). The increased rate also changes the absolute abundances. The mass fraction of the 160 increases by a factor of 1.5 to 2 (for an increase in the rate a factor of 3 and 5 , respectively) for models with mass in the range 10 to $30 \mathrm{M}_{\Theta}$ (Thielemann, 1984). This raises the possibility for an interesting constraint. Stars of about $20 \mathrm{M}_{\odot}$ are argued to be typical agents of nucleosynthesis since they are near the peak of the curve weighting ejected mass with the stellar mass function. Twarog and Wheeler (1982) pointed out, however, that for standard yields and mass functions, there was a danger that if all the massive stars $\left(M>12 \mathrm{M}_{\odot}\right)$ exploded, an overproduction of various elements, particularly oxygen, would occur. The overproduction was at least a factor of 1.5 for a reasonably steep mass function $\left(\mathrm{dN} / \mathrm{dM} \propto \mathrm{M}^{-3}\right)$, and worse for shallower mass functions which imply greater numbers of massive stars. With the new rates, the overproduction would be at least a factor of 2 to 3 , and that is far larger than the scatter in the observed values at the current epoch.

There are three possible conclusions that might be reached from this discussion. Perhaps the rate for ${ }^{12} \mathrm{C}(\alpha, \gamma){ }^{16} \mathrm{O}$ is not enhanced. This helps the overproduction of oxygen, but restores old problems with the ratios of certain species. For instance $\mathrm{Ne} / \mathrm{O}$ and $\mathrm{Mg} / \mathrm{O}$ are typically too high by a factor of about four. A second possibility is that the rate is higher and the bulk production of elements like oxygen is predicted to be too large. This may imply that some, perhaps a majority, of the massive stars that were presumed to be the major source of nucleosynthesis collapse rather than explode. Alternatively, the 
excess ejecta may be expelled from the Galaxy, a notion previously raised for SNI. A third possibility is that we simply do not yet know the nuclear physics and the evolution of massive stars sufficiently well to make precise comparisons with observations. In this case, any conclusion concerning the fate of massive stars, that they explode and provide the site of synthesis of heavy elements, or that they collapse and do not, is premature. The fate of Wolf-Rayet stars which are thought to be representative of this class of massive stars is likewise uncertain.

\section{Supernova Classifications}

Recent developments have suggested we are on the verge of a period of new understanding and reclassification of supernovae. Despite the suggestion of five classes by Zwicky (1965), supernovae have been typically characterized by their spectra as either SNI (no apparent hydrogen) thought to be caused by a thermonuclear explosion or SNII (with detectable hydrogen) thought to arise from core collapse. Two recent supernovae, SN 1983n and SN19841, have confirmed that there is a class of supernovae which blur the typical lines of empirical classification and provoke new questions about the nature of supernovae.

SN1983n and SN19841 had spectra which were virtually identical at maximum light (Wheeler and Levreault 1985). They are hydrogen deficient, and certainly do not resemble the spectra of SNII (nor any other of Zwicky's types). The spectra qualitatively resemble those of SNI, but there are distinct differences, and hence these events have been called peculiar SNI. The Doppler broadening and hence the ejecta velocity is about the same as for a SNI. The shape of the light curve is also very similar. This implies that the mass of the ejecta, which determines the thermal diffusion time and the width of the light curve peak, is comparable for the classical and peculiar SNI. The peculiar events seem to be associated with Population I stellar environments, HII regions, spiral arms, and bars of barred spiral galaxies, environments distinctly eschewed by classical SNI. SN1983n was a radio supernova (Sramek et al 1984) and SN19841 may be as well (Sramek 1985). No classical SNI has been observed in the radio.

The peculiar SNI appear to be dimmer at maximum light than the classical variety by a factor of order four. In the absence of a hydrogen envelope, the luminosity is presumed to come from the radioactive decay of $56 \mathrm{Ni}$, as for classical SNI. Less luminosity implies less nickel ejected, however, and hence a weaker thermonuclear explosion. Since the peculiar events seem to have the same mass and velocity and hence kinetic energy as the classical variety, the implication is that the kinetic energy can not arise purely from thermonuclear burning, but must rely on some other process. The only other obvious candidate is core collapse, as for SNII.

The ejecta mass must be of order $2-3 \mathrm{M}_{\odot}$ from the similarity of the light curve shapes. If to the ejecta mass one adds the mass of a neutron star, of order $1 \mathrm{M}_{\odot}$, then the mass of the immediate progenitor of the peculiar SNI is of order 3- $4 \mathrm{M}_{\odot}$. If this is interpreted as the mass of an evolving helium core, then the original main sequence mass would be of order $10-20 \mathrm{M}_{\odot}$, basically the same mass range as that which produces the bulk of the SNII. This implies that while the true nature of the peculiar SNI is still uncertain, there is a strong suggestion that the physical mechanism of their explosion is more like that of a SNII than a SNI, despite the spectral similarity to the latter. The relatively small mass deduced for the progenitor stars argues that the peculiar SNI are not directly related to Wolf-Rayet stars despite the suggestive nature of the hydrogen deficiency and the association with Pop I. Note, however, that Cahen (1985) argues that 
Wolf-Rayet stars can be as bright as normal supernovae despite their lack of extended envelopes if they eject of order $0.1 \mathrm{M}_{\odot}$ of $56 \mathrm{Ni}$, and that the light curve decline is enhanced by the rapid recombination wave in the helium envelope.

SNII also come in two categories, characterized by their light curves, those which show a plateau, and those which show a linear decline. Recently Doggett and Branch (1985) have noted that whereas the majority ( $2 / 3$ of the sample) plateau events are about $1.5 \mathrm{~m}$ dimmer than SNI, the linear events (1/3 of the sample) are nearly as bright, and that when plotted on the same scale, the light curves of the linear SNII qualitatively resemble those of classical SNI. This leads to the following speculation. Perhaps of the events which show hydrogen in the spectrum the plateau SNII are core collapse events from stars with $\mathrm{M}>8 \mathrm{M}_{\odot}$ and the linear variety are the result of thermonuclear explosions of stars with degenerate $C / O$ cores and mass slightly less than $8 \mathrm{M}_{\odot}$. Of the supernovae with no apparent hydrogen, the classical SNI are thermonuclear explosions of $\mathrm{C} / \mathrm{O}$ cores which have been laid bare in binary systems and then rekindled, whereas the peculiar events arise from the collapse of more massive stars which have lost their envelopes by mass transfer or winds.

\section{Conclusion}

There is no question that some supernovae come from luminous stars. The question of which luminous stars produce supernovae is more difficult to answer. Statistics suggest that stars with $M>10 M_{\Theta}$ (or perhaps a little less) produce SNII. Current evolutionary theory demands that stars above this limit collapse to form neutron stars, but the question of the progenitors of pulsars and of SNR of various morphology is still very uncertain. Some galaxies with an abundance of luminous stars nevertheless seem not to produce a corresponding number of SNII. Constraints from nucleosynthesis hint that not all massive stars should eject their full complement of heavy elements. Some may collapse to produce black holes or evolve in ways or environments we have yet to fathom.

This research was supported in part by NSF grant 8413301 .

\section{References}

Cahen, S. 1986, Proceedings of the Fifth Moriond Conference on Astrophysics.

Campbell, A. W., and Terlevich, R. 1984, MNRAS, 211, 15.

Doggett, J. B., and Branch, D. 1985, preprint.

Falk, S. W., and Arnett, W. D. 1977, Astrophys. J. Suppl., 33, 515.

Helfand, D. J. 1984a, in Supernova Remnants and Their X-Ray Emission. ed. P. Gorenstein and J. Danziger (Dordrecht: Reidel).

Helfand, D. J. 1984b, private communication.

Hillebrandt, W., Nomoto, K. and Wolff, R. G. 1984, Astr, and Ap., 133, 175.

Kettner, K. U., Becker, H. W., Buchmann, L., Görres, J., Kräwinkel, H., Rolfs, C., Schmalbrock, P., Trautvetter, H. P., Vlieks, A. 1982, Z. Physik, A308, 73.

Kronberg, P. P., Biermann, P., and Schwab, F. R. 1985, Ap. J. 291, 693.

Li, Z., Wheeler, J. C., and Bash, F. N. 1984, in Proceedings of the Erice Workshop on Stellar Nucleosynthesis ed. C. Chiosi and A. Renzini.

Li, Z., Wheeler, J. C., Bash, F. N., and Jefferys, W. H. 1985, in preparation.

Litvinova, I. Yu., and Nadyoozhin, D. K. 1983, Ap. and Space Sci., 89, 89. 
Lyne, A. G. 1982, in Supernovae: A Survey of Current Research, ed. M. J. Rees and R. J. Stoneham (Dordrecht: Reidel), p. 405.

Mazurek, T. J., Meier, D. L., and Wheeler, J. C. 1977, Astrophys. J, 215, 518.

Nomoto, K. 1984, in Proceedings of the Erice Workshop on Stellar Nucleosynthesis, ed. C. Chiosi and A. Renzini, in press.

Oemler, A., and Tinsley, B. M. 1979, A. J., 84, 985.

Sramek, R. A., Panagia, N., and Weiler, K. W. 1984, Ap. J. Letters, 285, L63.

Sramek, R. A. 1985, private communication.

Sutherland, P. G., and Wheeler, J. C. 1984, Ap. J., 280, 282.

Tammann, G. A. 1982, in Supernovae: A Survey of Current Research, ed. M. J. Rees and R. J. Stoneham (Dordrecht: Reidel), p. 371.

Thielemann, F. K. 1984, private communication.

Twarog, B. A., and Wheeler, J. C. 1982, Ap. J. 261, 636.

Wheeler, J. C. 1977, Astrophys. Space Sci., 50, 125.

Wheeler, J. C. 1981, Reports on Progress in Physics, 44, 85.

Wheeler, J. C. and Levereault, R. 1985, Ap. J. Letters, in press.

Wheeler, J. C., and Wheeler, J. A. 1983, in Science Underground, ed. M. M. Nieto, W. C. Haxton, C. M. Hoffman, E. W. Kolb, V. D. Sandberg, and J. W. Toevs (New York: American Institute of Physics), p. 214.

Wilson, J. R. 1984, preprint.

Woosley, S. E. 1986, Proceedings of the Fifth Moriond Conference on Astrophysics.

Woosley, S. E., and Weaver, T. A. 1982, in Supernovae: A Survey of Current Research, ed. M. J. Rees and R. J. Stoneham (Dordrecht: Reidel).

Zwicky, F. 1965, in Stars and Stellar Systems, ed. L. H. Aller and D. B. McLaughlin (Chicago, University of Chicago Press), p. 367, vol. VIII.

Discussion : WHEELER.

FEAST :

Do you believe that the statistics of runaway stars gives one any information on the masses of supernovae?

WHEELER :

I have never been convinced that runaway $O$, $B$ stars had anything to do with supernovae. The recent work of Gies, referred to by de Loore, does nothing to dissuade me of that notion. 


\section{SREENIVASAN :}

Is there a good theroretical reason why WR stars should expell more than $2-3 M_{0}$ in a $S N$ event?

\section{WHEELER :}

Observations suggest that Wolf-Rayet stars have masses exceeding $8 \mathrm{M}$. I believe the theory is consistent with that, but it is possible that a final very rapid, very short lived mass loss phase could bring the nass down.

\section{DE JAGER :}

1) Theoretical research seems to show that Wolf-Rayet stars become supernovae. But in view of the low birth rate of WR's the WR supernovae must constitute a small fraction of the supernovae. Since WR's are H-poor they cannot yield type II SN's, but they cannot be type I either because WR's are extreme population I objects. So, WR supernovae, if they exist, constitute another class of fairly rare supernovae.

With regard to Niemela's interesting observations $I$ think it is not sure that the progenitor was a WR; the WR characteristics may just have been a consequence of the explosion just having started.

2 ) the Cas A progenitor must have been faint at maximum, $M$ about - 12. How do we reconcile this with the claim that the progenitor was massive?

\section{WHEELER :}

As I said, I think there is doubt that all Wolf-Rayet stars necessarily explode even on the basis of the theoretical models. I argued that constraints based on nucleosynthesis may cut either way. Yodels for the core-collapse induced explosion are still ambivalent. One can understand why Cas $A$ may have been $-12^{\mathrm{m}}$ at peak in terms of an explosion in a rather compact object like a Wolf-Rayet star so that much of the shock energy is dissipated in adiabatic expansion before the ejecta become sufficiently optically thin to radiate, as has been argued by Chevalier. The work of Cahen, Schaeffer and Cassi et al. suggests that if about $0.1 \mathrm{M}$ of radioactive material is ejected, the event will have a normal Type If magnitude, so Cas $A$ must not have ejected such an ainount of nickel.

\section{CAMPBELL :}

I would just like to say something about NGC $5253.1 / \frac{1}{4}$ 's a lenticular galaxy with a smooth red population, well fit by an $R^{1 / 4}$ law, 
which has a starburst nucleus. Why not describe it as an so galaxy with a nuclear starburst, just as for spirals?

\section{SANDAGE :}

The morphological definition of an SO is that it doesn't have dust lanes or emission lines. NGC 5253 is amorphous like M82 and therefore should be classified as IrII.

\section{WHEELER :}

But it is not amorphous- for example, Jedrjewski's thesis shows that it is a lenticular except right at the nucleus.

\section{KAFATOS :}

Craig, could you tell us something about the number statistics of these peculiar type I supernovae?

\section{WHEELER :}

Over the last few years during which these SN have been observed the number seens to be about $10 \%$ of all supernovae. Branch has argued that they may be selected against because of the fainter magnitudes compared to classical type I events.

\section{GRAHAM to NIEMELA :}

Is it not true that in Niemela's work with Maria Teresa Ruiz and Mark Phillips they saw a WR type spectrum in a recent SN before maximum and does this not therefore support the idea that some WR stars turn into supernovae?

\section{NIEMELA :}

Yes, we observed a WN6 type spectrum about 10 days before the maximum light. However, we do not know whether this WR spectrum was caused by the explosion, or if it was the WN star that exploded. In any case, the spectrum we saw was $\mathrm{N}$ enriched (ref. Ap.J. 289, 52, 1985). 